\title{
Anisotropy-induced spin reorientation in chemically modulated amorphous ferrimagnetic films
}

\author{
E. Kirk, ${ }^{1,2}$ C. Bull $\odot,{ }^{3}$ S. Finizio $\odot,{ }^{2}$ H. Sepehri-Amin,${ }^{4}$ S. Wintz $\odot,{ }^{2}$ A. K. Suszka, ${ }^{1,2}$ N. S. Bingham,,${ }^{1,2}$ P. Warnicke, ${ }^{2}$ \\ K. Hono, ${ }^{4}$ P. W. Nutter, ${ }^{3}$ J. Raabe $\odot,{ }^{2}$ G. Hrkac, ${ }^{5, *}$ T. Thomson $\odot,{ }^{3, \dagger}$ and L. J. Heyderman $\oplus^{1,2}$ \\ ${ }^{1}$ Laboratory for Mesoscopic Systems, Department of Materials, ETH Zurich, 8093 Zurich, Switzerland \\ ${ }^{2}$ Paul Scherrer Institute, 5232 Villigen PSI, Switzerland \\ ${ }^{3}$ Department of Computer Science, University of Manchester, Oxford Road, Manchester M13 9PL, United Kingdom \\ ${ }^{4}$ Research Center for Magnetic and Spintronic Materials, National Institute for Materials Science, Tsukuba 305-0047, Japan \\ ${ }^{5}$ College of Engineering, Mathematics and Physical Sciences, University of Exeter, North Park Road, Exeter EX4 4QF, United Kingdom
}

(Received 27 February 2020; revised 20 April 2020; accepted 9 June 2020; published 7 July 2020)

\begin{abstract}
The ability to tune the competition between the in-plane and out-of-plane orientation of magnetization provides a means to construct thermal sensors with a sharp spin reorientation transition at specific temperatures. We have observed such a tuneable, temperature-driven spin reorientation in structurally amorphous, ferrimagnetic rare-earth transition-metal alloy thin films using scanning transmission x-ray microscopy and magnetic measurements. The nature of the spin reorientation transition in FeGd can be fully explained by a nonequilibrium, nanoscale modulation of the chemical composition of the films. This modulation leads to a magnetic domain pattern of nanoscale speckles superimposed on a background of in-plane domains that form Landau configurations in $\mu \mathrm{m}$-scale patterned elements. It is this speckle magnetic structure that gives rise to a sharp two-step reversal mechanism that is temperature dependent. The possibility to balance competing anisotropies through the temperature opens opportunities to create and manipulate topological spin textures.
\end{abstract}

DOI: 10.1103/PhysRevMaterials.4.074403

\section{INTRODUCTION}

Ferrimagnets, with two magnetically ordered sublattices that are coupled, offer opportunities to create a host of functional materials. A particularly fascinating class of these materials are amorphous rare-earth transition-metal (RE-TM) alloys in thin film form with perpendicular magnetic anisotropy [1]. Recently, RE-TM alloy thin films have garnered significant attention as materials for ultrafast, all-optical magnetic switching [2-4], as systems that can support topologically isolated structures such as Skyrmions [5-7] and as materials for spin-orbit torque devices [8]. In the past, their high perpendicular magnetic anisotropy and lack of grain boundaries have led them to be exploited for bubble memories [9] and used as magnetooptic recording media [10].

Particularly interesting is the ability to design RE-TM thin films where temperature can be used to control spin reorientation [11,12], saturation magnetization [13], magnetic anisotropy [14], and induce specific spin configurations [5]. Many RE-TM thin films have a strong perpendicular magnetic anisotropy, although the mechanism responsible for generating this anisotropy in nominally amorphous RE-TM thin films is still not fully explained with a number of competing hypotheses proposed for various RE-TM alloy thin films. Five possible mechanisms capable of inducing perpendicular anisotropy in amorphous RE-TM alloy thin films are typically considered: (i) dipolar interaction between atoms leading to

\footnotetext{
*Corresponding author: g.hrkac@exeter.ac.uk

†Corresponding author: thomas.thomson@manchester.ac.uk
}

pair ordering due to surface roughness [15], (ii) selective resputtering due to the different bonding strengths of RE and TM atoms leading to pair ordering [16], (iii) rare-earth single-ion anisotropy [17], (iv) magnetoelastic coupling leading to bond-orientation anisotropy $[18,19]$, and (v) induced microstructural changes [20], with perhaps the most widely accepted being that of Harris et al. [21] who demonstrated anisotropic pair-pair correlations in TbFe using EXAFS similar to mechanism (ii). Here we explore a further hypothesis, previously proposed by Graves et al. [22] for a similar class of GdFeCo thin films, which is that of chemical phase separation. Indeed, the chemical phase separation is something that is frequently overlooked as the assumption is often made that amorphous films are homogeneous.

In this paper, we choose FeGd as a prototype RE-TM amorphous, ferrimagnetic thin film system with a composition tuneable compensation temperature [23-27]. Using scanning transmission $\mathrm{X}$-ray microscopy (STXM), vibrating sample magnetometer (VSM) and superconducting quantum interference device-VSM (SQUID-VSM) measurements we show that for an appropriately chosen composition, FeGd thin films support a spin reorientation transition. We demonstrate how the complex nanoscale composition of these films leads to a two-step hysteresis behavior at temperatures above the spin reorientation temperature. The intricate magnetic nature of these RE-TM alloys originates from a metastable thermodynamic equilibrium. In particular, complex spin dynamics and local changes in magnetic properties can be induced via temperature and/or fabrication processes. It should be mentioned that a spin reorientation on changing the temperature has previously been reported in ultrathin Fe films 
[28,29] and RE-TM alloy thin films [11,30,12]. However, the ability to control and tailor this transition, and the resulting macroscopic properties, including anisotropy and coercivity, sufficiently for use in devices such as sensors, requires a more detailed understanding of the relationship between the evolution of the spin orientation with temperature and the thickness-dependent nanoscale compositional structure. Here we provide a detailed explanation of the influence of the chemical composition on the existence and temperature dependence of a spin reorientation transition in these materials.

\section{EXPERIMENTAL}

\section{A. Sample preparation}

We prepared $\mathrm{FeGd}(t \mathrm{~nm}) / \mathrm{Ta}(8 \mathrm{~nm})$ films (where $t$ is the FeGd thickness), by dc magnetron cosputtering at room temperature from elemental $\mathrm{Fe}$ and Gd targets onto Si (100) substrates coated with a $95 \mathrm{~nm}$ LPCVD grown $\mathrm{Si}_{3} \mathrm{~N}_{4}$ layer. The two thicknesses of film were sputtered under identical conditions resulting in an average composition of $\mathrm{Fe}_{0.69} \mathrm{Gd}_{0.31}$ at $\%$. The composition was chosen to ensure that the spin reorientation occurred below room temperature thereby eliminating any possibility of recrystallization due to elevated measurement temperatures. The base pressure of the loadlocked sputter chamber was better than $3.0 \times 10^{-9} \mathrm{mBar}$ and the Ar sputtering gas pressure was $4.8 \times 10^{-3} \mathrm{mBar}$. Films with thicknesses of $t=20 \mathrm{~nm}$ and $t=40 \mathrm{~nm}$ were produced to represent the two key regimes in the evolution of the observed lateral chemical segregation. STXM measurements were undertaken on the $40 \mathrm{~nm}$ thick films patterned into arrays of square-shaped microstructured magnetic elements with side length $5 \mu \mathrm{m}$. For the patterning MMA/PMMA bilayer lift-off masks were spin coated on $1 \mathrm{~mm} \times 1 \mathrm{~mm} \mathrm{Si}_{3} \mathrm{~N}_{4}$ membranes, where the membrane thickness of $200 \mathrm{~nm}$ was chosen to allow the transmission of $x$ rays. After deposition of the films, lift off was performed in acetone. The separation of the square-shaped microstructures was sufficiently large to ensure that the influence of stray fields from neighboring squares was negligible. Continuous film samples were used for the magnetometry measurements.

\section{B. Measurements}

Magnetic measurements were performed using a Quantum Design MPMS3 SQUID-VSM magnetometer. The films were mounted on a quartz rod holder with the magnetic field applied in the sample plane. To characterize the spin reorientation, temperature-dependent $\mathrm{M}(\mathrm{H})$ loops were measured with a maximum applied magnetic field of $4 \mathrm{kOe}$, which was sufficient to ensure that all switching events had taken place. For $T=180 \mathrm{~K}$, as shown in Supplemental Material Fig. S1 [31], there is an abrupt reversal, which is associated with magnetization reversal along the in-plane (IP) easy axis. For temperatures above the spin reorientation transition, the easy axis becomes out of plane (OOP). Therefore, the $\mathrm{M}(\mathrm{H})$ measurements acquire the characteristics of a hard axis loop, with a slow increase in magnetization to saturation indicating a rotation of the magnetization, as shown by the $T=275 \mathrm{~K}$ data in Supplemental Material Fig. S1 [31]. At intermediate temperatures $\mathrm{M}(\mathrm{H})$ loops consist of a superposition of these two behaviors, and from these loops each component of magnetization can be estimated. Hence three magnetization values can be obtained, the total magnetization measured at $4 \mathrm{kOe}$, the in-plane magnetization and the out-of-plane magnetization. The difference in $\mathrm{M}(\mathrm{H})$ behavior at different temperatures was confirmed by high-field $\mathrm{M}(\mathrm{H})$ measurements where the magnetic field was increased up to $70 \mathrm{kOe}$, as well as vector VSM measurements.

$\mathrm{X}$-ray reflectivity (XRR) and perpendicular X-ray diffraction (XRD) measurements were undertaken using a Rigaku Smartlab X-Ray diffractometer, operating at the $\mathrm{CuK} \alpha 1$ wavelength, $\lambda=1.540593(2) \AA$. XRR measurements were taken using a step size of $0.0004^{\circ}$ over a two-theta range of $0.1-7.0^{\circ}$ at rate of $0.01^{\circ} /$ minute. For XRD measurements, a $\mathrm{Ge}(220)$ double bounce monochromator was used to measure diffraction spectra with a step size of $0.02^{\circ}$, over a twotheta range of $20.0-80.0^{\circ}$ at a rate of $0.1^{\circ} / \mathrm{min}$. Measured reflectivity data was fitted to a simulated curve generated from a defined structural model of the thin film using the Parrett recursive formalism [32] implemented in the GenX reflectivity package [33] in order to obtain the depth-dependent sample structure. The scattering length density (SLD) profile from this model was parametrized to obtain the film thickness, interfacial root mean square (RMS) roughness and density (SLD) values for individual layers in each sample.

Scanning transmission electron microscopy (STEM) was performed using a Titan G2 80-200 microscope with a probe aberration corrector. The specimens for the STEM analysis were prepared using an FEI Helios G4-UX dual-beam system with the lift out method. The distribution of constituent elements and chemical composition of the films were measured using energy-dispersive x-ray spectroscopy (EDS).

Scanning transmission $\mathrm{x}$-ray microscopy (STXM) measurements were undertaken at the PolLux beamline (X07DA), Swiss Light Source, Paul Scherrer Institute [34] on membrane samples. Magnetic contrast was obtained using x-ray magnetic circular dichroism (XMCD) that results from a differential absorption of circularly polarized $\mathrm{x}$ rays for magnetization parallel or antiparallel to the beam propagation direction. The specimens were imaged both in the normal incidence geometry, sensitive to OOP magnetization, and with the sample at an angle of $30^{\circ}$ to the beam. For an angle of $30^{\circ}$, the components of magnetization in the domains with both the OOP and IP magnetization could be imaged. Images were taken with the $\mathrm{X}$ ray energy tuned to the $\mathrm{Gd} \mathrm{M}_{5}$ absorption edge at $1190 \mathrm{eV}$. A series of images of a $5 \mu \mathrm{m}$ square-shaped FeGd element were recorded at temperatures between $150 \mathrm{~K}$ and $240 \mathrm{~K}$, which spans the temperature range over which the spin reorientation transition took place.

\section{RESULTS AND DISCUSSION}

The temperature dependence of the spin orientation in the two FeGd RE-TM thin films is shown in Figs. 1(a) and (b). The data were obtained from in-plane $\mathrm{M}(\mathrm{H})$ loops measured by SQUID-VSM magnetometry over the temperature range $150-300 \mathrm{~K}$ with a maximum applied field of $4 \mathrm{kOe}$ as described in the Sec. II B. This data shows that manipulation of the spin reorientation temperature is possible in FeGd thin films. In particular, it can be seen that the spin reorientation 

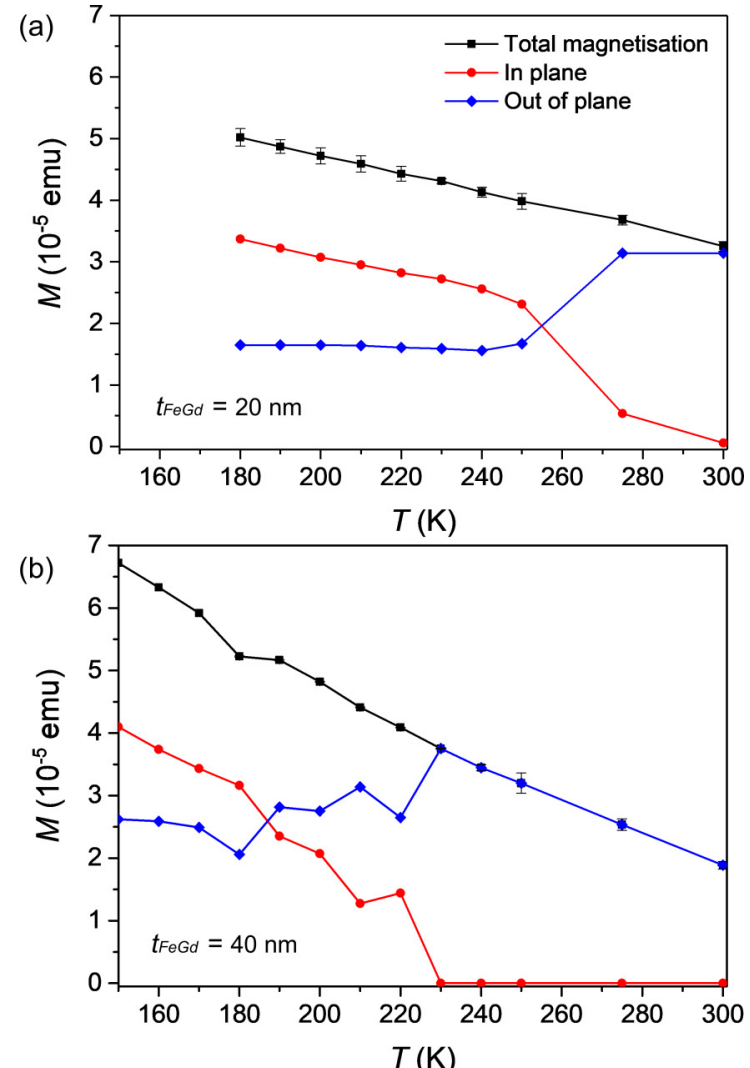

(c)

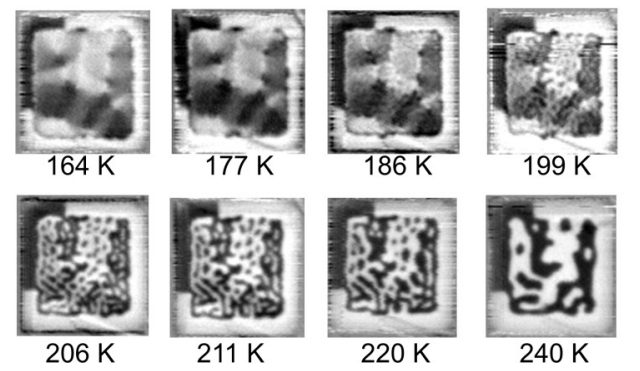

FIG. 1. IP Magnetization (red circle), OOP magnetization (blue diamond), and total magnetization (black square) vs temperature for (a) $20 \mathrm{~nm}$, (b) $40 \mathrm{~nm}$ FeGd continuous films, and (c) STXM images of a $40 \mathrm{~nm}$-thick, $5 \times 5 \mu^{2}$ square-shaped FeGd microstructure on a membrane substrate, which show the transition from IP to OOP magnetization. The total image size is approximately $6 \mu \mathrm{m}$ square.

starts at $250 \mathrm{~K}$ for the $20 \mathrm{~nm}$ film and shifts towards a lower temperature of $190 \mathrm{~K}$ for the $40 \mathrm{~nm}$ film. The temperature dependence of magnetization for both samples, measured using an applied field of $70 \mathrm{kOe}$, is shown in the Supplemental Material, Fig. S2 [31]. These data demonstrate a similar behavior in saturation magnetization (Ms) vs temperature $(T)$ for both samples. The value of Ms shows some variation between samples, which, given the identical sputtering conditions employed, is unexpected and most likely reflects the sensitivity of the system to chemical inhomogeneities. In order to clarify the nature of the spin reorientation, XMCD-STXM images of the magnetic configuration of FeGd elements with $40 \mathrm{~nm}$ thickness were recorded. The STXM images presented in Fig. 1(c) were taken with the sample normal oriented at $30^{\circ}$ to the x-ray beam so that both in-plane and out-of-plane components of the magnetization can be observed. The pixel size of the STXM images is $\approx 50 \mathrm{~nm}$ providing nanoscale observations of the evolution of the domain structure during the spin reorientation transition. Specifically, at $164 \mathrm{~K}$, $\mu \mathrm{m}$-sized in-plane domains with vortices are present in the films. The persistent OOP frame around each of the FeGd microstructures was observed for all FeGd samples measured and is likely to be related to Gd enrichment, as indicated by XAS measurements, under the deep lithographic lift-off edge. This boundary region did not affect the STXM data obtained from the FeGd microstructures. In addition, all other measurements were undertaken on continuous films and are therefore unaffected by this boundary region. As the temperature is increased to $177 \mathrm{~K}$, a fine domain structure consisting of small domains starts to emerge. We estimate the diameter of these domains to be between 100 and $150 \mathrm{~nm}$ but note there is a significant uncertainty in this determination, see Supplemental Material Fig. S3 and discussion [31]. At 186 K, fine scale OOP domains are observed to nucleate homogeneously within the IP domains. The IP structure of $\mu \mathrm{m}$-sized domains and vortices continues to coexist alongside the fine scale OOP domain structures at $199 \mathrm{~K}$, with the direction of magnetization in the new OOP domains observed to be strongly correlated to the direction of magnetization in the previous IP domains. In particular, the grayscale contrast associated with circulation of the IP magnetization around vortex cores is completely replaced by the black/white contrast of OOP domains at $211 \mathrm{~K}$. Further increasing the temperature leads to large connected OOP domains above $240 \mathrm{~K}$. This interpretation of the temperature evolution of the domain structure was confirmed by additional measurements taken in the purely out-of-plane geometry, Supplemental Material, Fig. S4 [31].

The observed spin reorientation can be understood in terms of a competition between the magnetic energy contributions. In the case of homogeneous films, the transition from the IP to OOP spin configuration is a function of the competition between demagnetization energy (volume and shape anisotropy), magnetic anisotropy energy, and exchange energy. In a magnetic hybrid structure, where one encounters two or more different regions with different magnetic properties, more complex spin configurations can occur. The underlying phenomenon responsible for the complex magnetic configurations, as seen in Fig. 1(c), is competing energies on a local level, such as localized changes in magnetic anisotropy or variations in the magnetization resulting from an inhomogeneous chemical composition.

To fully understand the details of the observed magnetic behavior we adopted an approach combining microstructural analysis and micromagnetic simulation. First, we undertook comprehensive measurements of the microstructural properties of the films using $\mathrm{x}$-ray diffraction (XRD) and transmission electron microscopy (TEM). These data, shown in Supplemental Material Fig. S5 [31], demonstrate that the films are structurally amorphous, both locally as determined by TEM, and over larger length scales as shown by XRD. Therefore, a mechanism such as the pseudocrystalline short-range order, proposed by Onton et al. [35] to describe the broadening of low-energy XRD peaks, is not responsible for the perpendicular magnetic anisotropy observed in our films. We find instead that the underlying phenomena is that of chemical segregation 

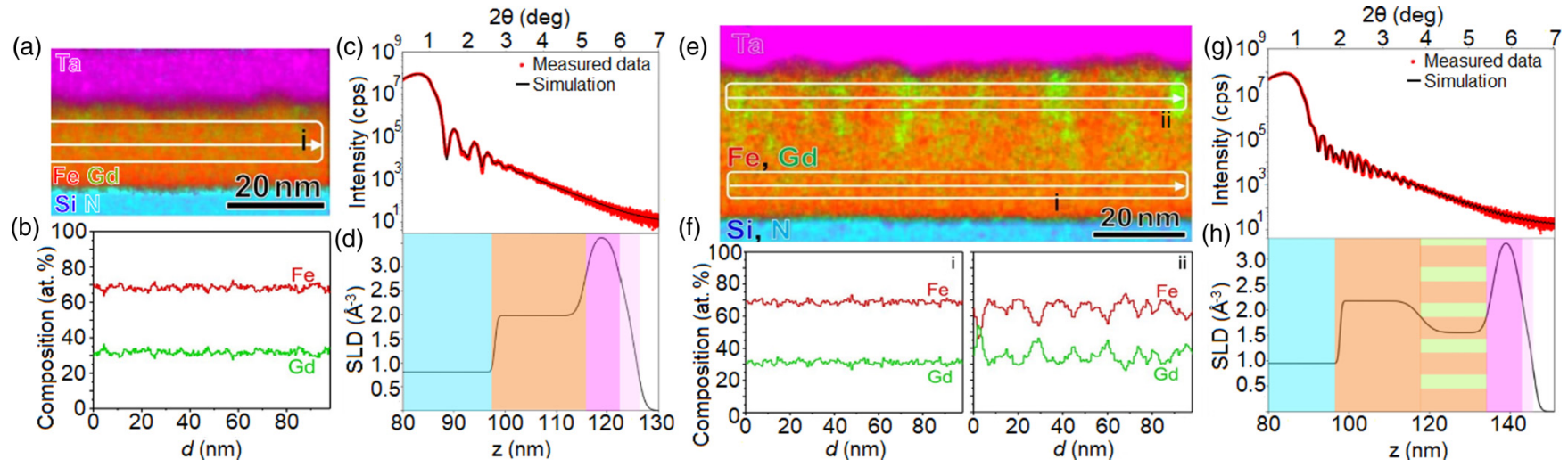

FIG. 2. (a-d) data for $20 \mathrm{~nm}$ FeGd film with (a) STEM-EDS map of constituent elements and (b) composition profile along the line in (a), (c) XRR data and simulation and (d) the corresponding scattering length density data. Data for the $40 \mathrm{~nm}$ FeGd film is shown in (e-h), with (e) STEM-EDS map, (f) composition line profiles obtained from lines marked with i and ii in (e), and (g) XRR data and simulation with (h) the corresponding scattering length density data.

as revealed by scanning transmission electron microscopy equipped with energy dispersive x-ray spectroscopy (STEMEDS) and x-ray reflectivity (XRR) measurements (see methods). Chemical mapping of cross-sectional STEM-EDS for both the $20 \mathrm{~nm}$ and $40 \mathrm{~nm}$ samples is shown in Figs. 2(a), 2(b), 2(e), and 2(f), and the measured and simulated XRR data with the corresponding scattering length density (SLD) data are shown in Figs. 2(c), 2(d), 2(g), and 2(h). The experimental data sets could be successfully simulated using four- and five-layer models as shown in Figs. 2(c), 2(d), and 2(g), 2(h), respectively, with numerical values obtained from the fits given in the Supplemental Material, Table SI [31]. Inclusion in the model of an oxidized Ta layer at the surface was essential to accurately reproduce the critical edge scattering in all samples investigated. The XRR scattering length density (SLD) data clearly demonstrate a change in density through the thickness of the film, as demonstrated in Figs. 2(d) and 2(h). The STEM-EDS data provide further insight; the STEM results do not provide any evidence of induced voids [36,37], which could potentially account for the density variation determined from the XRR analysis. The STEM-EDS maps show a compositional segregation occurs in the $40 \mathrm{~nm}$ film, which is absent in the $20 \mathrm{~nm}$ film. In the case of the $40 \mathrm{~nm}$ film, in the first $20 \mathrm{~nm}$ above the substrate, there is a uniform composition distribution with 68.8 at\% $\mathrm{Fe}$ and 31.2 at \% Gd, which is close to the nominal composition of the films [Fig. 2(f i)]. The standard deviation in the composition is 1.4 at \%. However, a periodic modulation in chemical composition emerges as the film thickness increases as is evident from Fig. 2(f ii), which is reflected by the fact that the standard deviation increases to 4.8 at \%. This segregation is accompanied by a small change in the average composition, which increases from $\mathrm{Gd}=31.2$ at $\%$ to $\mathrm{Gd}=35.4$ at \% with a commensurate reduction in Fe concentration, which is possibly due to the diffusion of $\mathrm{Fe}$ into $\mathrm{Ta}$ at the interfacial region. The chemical segregation leads to phase separation of ferrimagnetic properties, which is due to increased antiferromagnetic pair bonds between $\mathrm{Gd}-$ $\mathrm{Fe}$ relative to $\mathrm{Fe}-\mathrm{Fe}$ pair bonds. This compositional change with thickness provides a convincing explanation for the change in scattering length density determined from XRR measurements. In addition, the chemically induced columnar structure, seen in the periodic variation in the cross-sectional STEM images, leads to a magnetic easy axis parallel to the columns enriched with $\mathrm{Gd}$ embedded in an Fe-rich matrix due to the induced shape anisotropy. Together, these data clearly show a chemical phase separation. Interestingly, this induced phase separation and the associated preference for perpendicular magnetization only becomes established above a film thickness of $20 \mathrm{~nm}$. Given the time to deposit $20 \mathrm{~nm}$ of $\mathrm{FeGd}(10 \mathrm{~min})$ it is possible to speculate that this is a result of the gradual temperature increase that occurs naturally during sputtering.

As can be seen from the SQUID-VSM and STXM measurements in Fig. 1, the chemical separation has an effect on the spin reorientation temperature with a clear reduction for the $40 \mathrm{~nm}$ film. To clarify the quantitative and qualitative effects of this chemical segregation, we measured out-of-plane hysteresis loops of the $20 \mathrm{~nm}$ and $40 \mathrm{~nm} \mathrm{FeGd} \mathrm{films} \mathrm{at} 295$ $\mathrm{K}$ [see Fig. 3(b)], which shows that the magnetization is now perpendicular to the film plane. As expected, for the $20 \mathrm{~nm}$ film there is a single-step reversal behavior with a coercivity of $15 \mathrm{Oe}$, which is associated with the perpendicular anisotropy due to anisotropic pair-pair correlations. For the $40 \mathrm{~nm}$ film, the hysteresis loop is more complex with a two-step reversal. Inferring the behavior from the STEM-EDS and STXM measurements, we associate the second reversal step with the switching of the columnar structure, which gives rise to the formation of the speckled domain structure seen in Fig. 1(c).

To test the hypothesis that the point domain structure is responsible for the two-step reversal, we performed micromagnetic simulations, implementing a three-dimensional finite element / boundary element micromagnetic columnar model based on the measured composition line profiles as shown in Fig. 3(a), which allowed construction of a multiphase FEM model. The effective structure in the $40 \mathrm{~nm}$ film consists of a $20 \mathrm{~nm}$ thick layer of $\mathrm{Fe}_{2} \mathrm{Gd}$ and, on top of that, a $20 \mathrm{~nm}$ complex columnar structure. Although we constructed a columnar model specifically from the line profile, it should be noted that a simplified cylindrical column model with equal spacing also reproduces the two-step reversal behaviour. The difference in coercivity values is explained by the difference in the size of the simulated model and the experimental sample. 
(a)
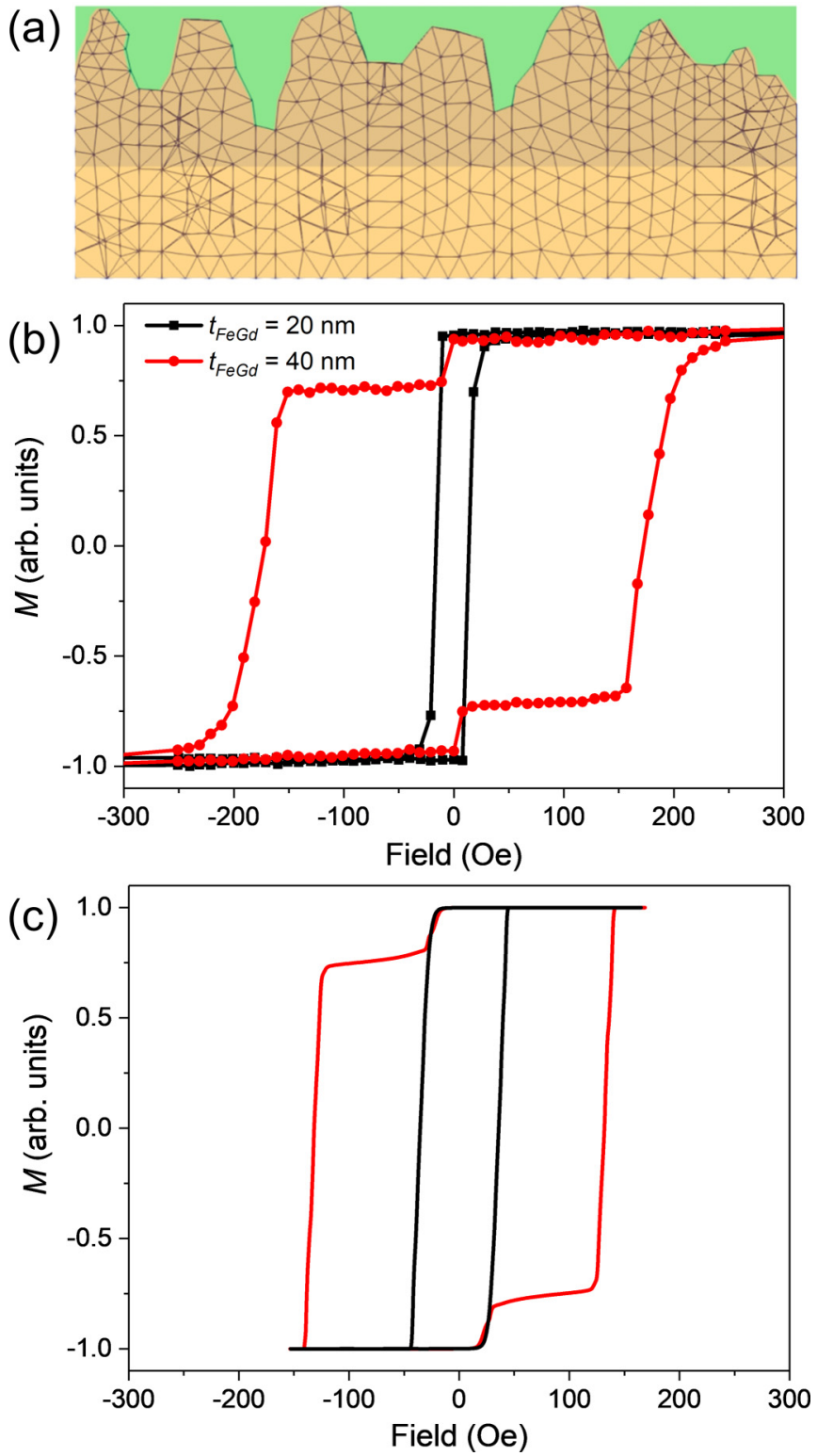

FIG. 3. (a) Illustration of micromagnetic model with mesh generated from STEM-EDS results, (b) room temperature hysteresis loops measured using a VSM for $40 \mathrm{~nm}$ and $20 \mathrm{~nm}$ FeGd film, and (c) simulations of $40 \mathrm{~nm}$ and $20 \mathrm{~nm}$ FeGd film demonstrating the same behavior as the measured loops.

The material parameters used in the micromagnetic simulations are $\mathrm{Ms}\left(\mathrm{Fe}_{2} \mathrm{Gd}\right)=0.369 \mathrm{~T}, \mathrm{Ms}(\mathrm{FeGd})=0.168 \mathrm{~T}, \mathrm{~A}=$ $1.0 \mathrm{e}^{-12} \mathrm{~J} / \mathrm{m}$ and are based on the calculation of the total Gibbs free energy [38].

Simulations of the hysteresis loops at room temperature [Fig. 3(c)], reproduce the two-step reversal seen in the experimental data in Fig. 3(b). Indeed, without this chemically segregated columnar structure, the simulations fail to reproduce the two-step reversal observed experimentally. Above the spin reorientation temperature, two distinct hysteretic behaviors are observed; a single-step loop with a coercivity of 15 Oe for the $20 \mathrm{~nm}$ and a two-step loop with coercivities of $5 \mathrm{Oe}$ and 170 Oe for the $40 \mathrm{~nm}$ film.

\section{CONCLUSIONS}

We have deduced the origin of the speckled domain magnetization pattern observed in the STXM images. In particular, we have shown using STEM-EDS and XRR that chemical segregation leads to the emergence of a columnar anisotropy in Gd-rich regions, which gives rise to the speckled domain structure and this is the driving force for the domain evolution in the $40 \mathrm{~nm}$ thick film. This secondary anisotropy phase, associated with the Gd-rich columns, lowers the spin reorientation temperature. The presence of the columns also narrows the temperature range over which the spin reorientation transition occurs, demonstrating the possibility to control spin reorientation transition temperature by manipulating the composition and microstructure. In this way, we achieve a higher functionality ferrimagnetic material that not only can be used as a soft ferrimagnetic reference sensor that exploits the first low-field transition, but also acts as a temperature sensor. Additionally, the ability to tailor the balance between IP and OOP anisotropies allows specific spin textures to form, opening exciting new possibilities for creating novel temperature-dependent Skyrmionic systems.

The data that support this study are available via the Zenodo repository [39].

\section{ACKNOWLEDGMENTS}

The authors wish to acknowledge Vitaliy Guzenko and Anja Weber at PSI, Switzerland for the electron beam lithography to pattern the lift-off mask for the arrays of microstructured squares on the membranes. We acknowledge the Paul Scherrer Institut, Villigen, Switzerland, for provision of synchrotron radiation beamtime at X07DA (PolLux). The authors also gratefully acknowledge the contribution of the facilities of the Henry Royce Institute through EPSRC Grants No. EP/S019367/1 and No. EP/P025021/1 for the x-ray reflectivity measurements.

E.K. and L.J.H. conceived the initial idea for project, with E.K., T.T., and G.H. taking overall responsibility for the project work. E.K. conceived the experiment, sputter deposited the FeGd films and performed the lithography to create the FeGd arrays. E.K. measured temperature-dependent $\mathrm{M}(\mathrm{H})$ loops and led the STXM and XMCD investigation of the temperature-dependent changes in the magnetic domain structure of FeGd film square-shaped microstructures. J.R. and S.F. were responsible for the PolLux beamline (X07DA) where the STXM measurements were made and contributed to the data processing and analysis. S.W., N.S.B., A.K.S., and P.W. also contributed to the STXM measurements. C.B. undertook the XRR measurements and data analysis. H.S.-A. and K.H. performed the TEM measurements and analysis. G.H. performed all the micromagnetic simulations. E.K., C.B., G.H., and T.T. were responsible for developing the analysis, explaining the results and coordinating the work. They were also responsible for drafting the paper to which all authors subsequently contributed. E.K., L.J.H., G.H., and T.T. completed the final form of the paper. 
[1] T. A. Ostler, R. F. L. Evans, R. W. Chantrell, U. Atxitia, O. Chubykalo-Fesenko, I. Radu, R. Abrudan, F. Radu, A. Tsukamoto, A. Itoh, A. Kirilyuk, T. Rasing, and A. Kimel, Crystallographically amorphous ferrimagnetic alloys: Comparing a localized atomistic spin model with experiments, Phys. Rev. B 84, 024407 (2011).

[2] S. Mangin, M. Gottwald, C.-H. Lambert, D. Steil, V. Uhlír, L. Pang, M. Hehn, S. Alebrand, M. Cinchetti, G. Malinowski, Y. Fainman, M. Aeschlimann, and E. E. Fullerton, Engineered materials for all-optical helicity-dependent magnetic switching, Nature Mater. 13, 286 (2014).

[3] C.-H. Lambert, S. Mangin, B. S. D. C. S. Varaprasad, Y. K. Takahashi, M. Hehn, M. Cinchetti, G. Malinowski, K. Hono, Y. Fainman, M. Aeschlimann, and E. E. Fullerton, All-optical control of ferromagnetic thin films and nanostructures, Science 345, 1337 (2014).

[4] C. D. Stanciu, F. Hansteen, A. V. Kimel, A. Kirilyuk, A. Tsukamoto, A. Itoh, and T. Rasing, All-Optical Magnetic Recording with Circularly Polarized Light, Phys. Rev. Lett. 99, 047601 (2007).

[5] J. C. T. Lee, J. J. Chess, S. A. Montoya, X. Shi, N. Tamura, S. K. Mishra, P. Fischer, B. J. McMorran, S. K. Sinha, E. E. Fullerton, S. D. Kevan, and S. Roy, Synthesizing skyrmion bound pairs in Fe-Gd thin films, Appl. Phys. Lett. 109, 022402 (2016).

[6] R. Streubel, C.-H. Lambert, N. Kent, P. Ercius, A. T. N'Diaye, C. Ophus, S. Salahuddin, and P. Fischer, Experimental Evidence of Chiral Ferrimagnetism in Amorphous GdCo Films, Adv. Mater. 30, 1800199 (2018).

[7] L. Caretta, M. Mann, F. Büttner, K. Ueda, B. Pfau, C. M. Günther, P. Hessing, A. Churikova, C. Klose, M. Schneider, D. Engel, C. Marcus, D. Bono, K. Bagschik, S. Eisebitt, and G. S. D. Beach, Fast current-driven domain walls and small skyrmions in a compensated ferrimagnet, Nature NanoTech. 13, 1154 (2018).

[8] N. Roschewsky, T. Matsumura, S. Cheema, F. Hellman, T. Kato, S. Iwata, and S. Salahuddin, Spin-orbit torques in ferrimagnetic GdFeCo alloys, Appl. Phys. Lett. 109, 112403 (2016).

[9] P. Chaudhari, J. J. Cuomo, and R. J. Gambino, Amorphous metallic films for bubble domain applications, IBM J. Res. Develop. 17, 66 (1973).

[10] S. Tsunashima, Magneto-optical recording, J. Phys. D: Appl. Phys. 34, R87 (2001).

[11] E. Stavrou, R. Sbiaa, and T. Suzuki, Different mechanisms of spin reorientation in exchange coupled double rare earthtransition metal layers with in-plane and perpendicular magnetic anisotropy, J. Appl. Phys. 87, 6893 (2000).

[12] W. He, H.-L. Liu, H.-Y. Wu, J.-W. Cai, and Z.-H. Cheng, Probing temperature-driven spin reorientation transition of GdFeCo film by Kerr loops and ferromagnetic resonance, Appl. Phys. Lett. 106, 042401 (2015).

[13] P. Hansen, C. Clausen, G. Much, M. Rosenkranz, and K. Witter, Magnetic and magneto-optlcal properties of rare earth transition metal alloys containing Gd, Tb, Fe, Co, J. Appl. Phys. 66, 756 (1989).

[14] F. Radu, R. Abrudan, I. Radu, D. Schmitz, and H. Zabel, Perpendicular exchange bias in ferrimagnetic spin valves, Nature Comms. 3, 715 (2012).

[15] H. Fu, M. Mansuripur, and P. Meystre, Generic Source of Perpendicular Anisotropy in Amorphous Rare-Earth-TransitionMetal Films, Phys. Rev. Lett. 66, 1086 (1991).
[16] R. J. Gambino and J. J. Cuomo, Selective resputtering-induced anisotropy in amorphous films, J. Vac. Sci. Technol. 15, 296 (1978).

[17] Y. Suzuki, S. Takayama, F. Kirino, and N. Ohta, Single ion model for perpendicular magnetic anisotropy in RE-TM amorphous films, IEEE Trans. Magn. 23, 2275 (1987).

[18] Y. Suzuki, J. Haimovich, and T. Egami, Bond-orientational anisotropy in metallic glasses observed by x-ray diffraction, Phys. Rev. B 35, 2162 (1987).

[19] X. Yan, M. Hirscher, T. Egami, and E. E. Marinero, Direct observation of anelastic bond-orientational anisotropy in amorphous $\mathrm{Tb}_{26} \mathrm{Fe}_{62} \mathrm{Co}_{2}$ thin films by x-ray diffraction, Phys. Rev B 43, 9300 (1991).

[20] D. Mergel, H. Heitmann, and P. Hansen, Pseudocrystalline model of the magnetic anisotropy in amorphous rare-earthtransition-metal, Phys. Rev. B 47, 882 (1993).

[21] V. G. Harris, K. D. Aylesworth, B. N. Das, W. T. Elam, and N. C. Koon, Structural Origins of Magnetic Anisotropy in Sputtered Amorphous Tb-Fe Films, Phys. Rev. Lett. 69, 1939 (1992).

[22] C. E. Graves, A. H. Reid, T. Wang, B. Wu, S. de Jong, K. Vahaplar, I. Radu, D. P. Bernstein, M. Messerschmidt, L. Müller, R. Coffee, M. Bionta, S. W. Epp, R. Hartmann, N. Kimmel, G. Hauser, A. Hartmann, P. Holl, H. Gorke, J. H. Mentink, A. Tsukamoto, A. Fognini, J. J. Turner, W. F. Schlotter, D. Rolles, H. Soltau, L. Strüder, Y. Acremann, A. V. Kimel, A. Kirilyuk, T. Rasing, J. Stöhr, A. O. Scherz, and H. A. Dürr, Nanoscale spin reversal by non-local angular momentum transfer following ultrafast laser excitation in ferrimagnetic GdFeCo, Nature Mater. 12, 293 (2013).

[23] J. Orehotsky and K. Schroder, Magnetic properties of amorphous $\mathrm{Fe}_{x} \mathrm{Gd}_{y}$ alloy thin films, J. Appl. Phys. 43, 2413 (1972).

[24] T. Morishita, Y. Togami, and K. Tsushima, Magnetism and structure of compositionally modulated $\mathrm{Fe}-\mathrm{Gd}$ thin films, J. Phys. Soc. Jpn. 54, 37 (1985).

[25] F. Hellman, E. N. Abarra, A. L. Shapiro, and R. B. van Dover, Specific heat of amorphous rare-earth-transition-metal films, Phys. Rev. B 58, 5672 (1998).

[26] Y. Mimura, N. Imamura, T. Kobayashi, A. Okada, and Y. Kushiro, Magnetic properties of amorphous alloy films of $\mathrm{Fe}$ with Gd, Tb, Dy, Ho, or Er, J. Appl. Phys. 49, 1208 (1978).

[27] H. Oezelt, E. Kirk, P. Wohlhuter, E. Muller, L. J. Heyderman, A. Kovacs, and T. Schrefl, Vortex motion in amorphous ferrimagnetic thin film elements, AIP Adv. 7, 056001 (2017).

[28] D. P. Pappas, K.-P. Kamper, and H. Hopster, Reversible Transition between Perpendicular and In-Plane Magnetization in Ultrathin Films, Phys. Rev. Lett. 64, 3179 (1990).

[29] A. Berger and H. Hopster, Nonequilibrium Magnetization near the Reorientation Phase Transition of Fe/Ag(100) Films, Phys. Rev. Lett. 76, 519 (1996).

[30] Y. Li, W. He, J.-W. Cai, and Z.-H. Cheng, Characterizing magnetization reversal processes of $\mathrm{GdFeCo}$ film in the vicinity of the spin reorientation transition temperature, J. Magn. Magn. Mater. 485, 369 (2019).

[31] See Supplemental Material at http://link.aps.org/supplemental/ 10.1103/PhysRevMaterials.4.074403 for additional magnetometry, STXM and structural data.

[32] L. G. Parratt, Surface studies of solida by total reflection of Xrays, Phys. Rev. 95, 359 (1954). 
[33] M. Björck and G. Andersson, GenX: an extensible X-ray reflectivity refinement program utilizing differential evolution, J. Appl. Crystallogr. 40, 1174 (2007).

[34] J. Raabe, G. Tzvetkov, U. Flechsig, M. Böge, A. Jaggi, B. Sarafimov, M. G. C. Vernooij, T. Huthwelker, H. Ade, D. Kilcoyne, T. Tyliszczak, R. H. Fink, and C. Quitmann, PolLux: A new facility for soft $x$-ray spectromicroscopy at the Swiss light source, Rev. Sci. Instrum. 79, 113704 (2008).

[35] A. Onton, N. Heiman, J. C. Suits, and W. Parrish, Structure and magnetic anisotropy of amorphous Gd-Co films, IBM J. Res. Develop. 20, 409 (1976).

[36] N. Honda, J. Ariake, K. Ouchi, and S.-i. Iwasaki, High coercivity in $\mathrm{Co}-\mathrm{Cr}$ film for perpendicular recording prepared by low temperature sputter-deposition, IEEE Trans. Magn. 30, 4023 (1994).

[37] G. Choe, Effect of film morphology on grain boundary segregation induced magnetic properties in heat treated CoCrPt/Cr films, IEEE Trans. Magn. 31, 2809 (1995).

[38] R. W. Chantrell, J. Fidler, T. Schrefl, and M. Wongsam, Micromagnetics: Finite Element Approach, in Encyclopedia of Materials: Science and Technology (Elsevier Science, Amsterdam, 2001), pp. 5651-5660.

[39] E. Kirk et al., dataset for Anisotropy-induced spin reorientation in chemically modulated amorphous ferrimagnetic films (2020), doi:10.5281/zenodo.3905875. 